\title{
New results on production matrices for geometric graphs
}

\author{
Guillermo Esteban Pascual, Clemens Huemer, Rodrigo I. Silveira ${ }^{1}$
}

Universitat Politècnica de Catalunya, Barcelona, Spain

\begin{abstract}
We present novel production matrices for non-crossing partitions, connected geometric graphs, and $k$-angulations, which provide another way of counting the number of such objects. For instance, a formula for the number of connected geometric graphs with given root degree, drawn on a set of $n$ points in convex position in the plane, is presented. Further, we find the characteristic polynomials and we provide a characterization of the eigenvectors of the production matrices.
\end{abstract}

Keywords: geometric graph, production matrix, Riordan array

\section{Introduction}

A geometric graph on a set of points $\mathcal{S}$ is a graph with vertex set $\mathcal{S}$ whose edges are straight-line segments with endpoints in $\mathcal{S}$. It is called non-crossing if no two edges intersect except at common endpoints. We focus on noncrossing geometric graphs on sets $\mathcal{S}$ of $n$ points in convex position. Numerous geometric graph classes exist, such as triangulations, connected graphs, or

1 Emails:

guillermo.esteban.pascual@estudiant.upc.edu, \{clemens.huemer, rodrigo.silveira\}@upc.edu 
spanning trees. A fundamental problem is to determine the number of graphs, for each class, as a function of $n$. Formulae were obtained for instance in [5]. Recently, in $[6,7,8]$, we showed how such geometric graphs can be counted by using an $n \times n$ matrix $A_{n}$, called production matrix, associated to each graph class. The number of these graphs for a fixed number of vertices is given by (a column of) a power of $A_{n}$. To derive a production matrix for a certain class, first the graphs on $i \leq n$ vertices are partitioned according to the degree of a specified root vertex (several definitions of degree are possible). Then each part is counted in the elements of an $n$-element vector $\boldsymbol{v}^{i}$, and hence the sum of the vector elements gives the number of geometric graphs for $i$ vertices. A production matrix $A_{n}$ is a matrix satisfying $\boldsymbol{v}^{i+1}=A_{n} \boldsymbol{v}^{i}$. Furthermore, an initial vector $\boldsymbol{v}^{c}$ containing the count for a constant number of vertices (often $\left.(1,0, \ldots, 0)^{\top}\right)$ is used, implying that $\boldsymbol{v}^{i+1}=A_{n}^{i+1-c} \boldsymbol{v}^{c}$. The method above to define $A_{n}$ based on a vertex degree, implicitly arranges graphs into a tree structure known as generating tree. In such a tree, for each graph on $i$ vertices and root degree $j$, the number of descendants on $i+1$ vertices with root degree $\ell$ (for each $\ell$ ) is known. Generating trees have been studied in several contexts, most notably for the ECO method [1], and for obtaining matrix representations of diverse combinatorial objects $[2,10]$.

Figure 1 shows production matrices for several graph classes, for $n=6$. We omit the general formula for the matrix entries. Matrix (a), see e.g. $[2,10]$, is used to count Catalan structures like triangulations or non-crossing partitions. We generalize matrix (a) to $k$-angulations, see matrices (e), (f). Matrix (e) is also a production matrix for spanning trees [6]. Instead of defining the degree of the root vertex $p_{n}$ as the number of its incident edges, we also define it as the number of vertices visible from a new vertex inserted between $p_{1}$ and $p_{n}$; two vertices are visible if the line segment connecting them does not intersect the interior of any edge of the graph. Matrices (b), (c), (d) are obtained using this definition. We point out that matrix (b) provides an alternative production matrix for Catalan structures and matrix (c) is a different matrix for geometric graphs, see [6]. For matrix (c) we use initial vector $(2,0, \ldots, 0)^{\top}$. As for matrix $(\mathrm{d})$, this is the first time that a production matrix for connected geometric graphs is derived, to the best of our knowledge. In addition to devising production matrices we deduce formulae for the vectors counting graphs of a given degree. For matrix (b), the vector that counts non-crossing partitions also counts special Dyck paths; compare with Table 2 in [3]. We then go on to study properties that have combinatorial implications. Among others, we obtain the characteristic polynomials of the matrices, and a characterization of the eigenvectors. Due to space constraints, we only sketch 


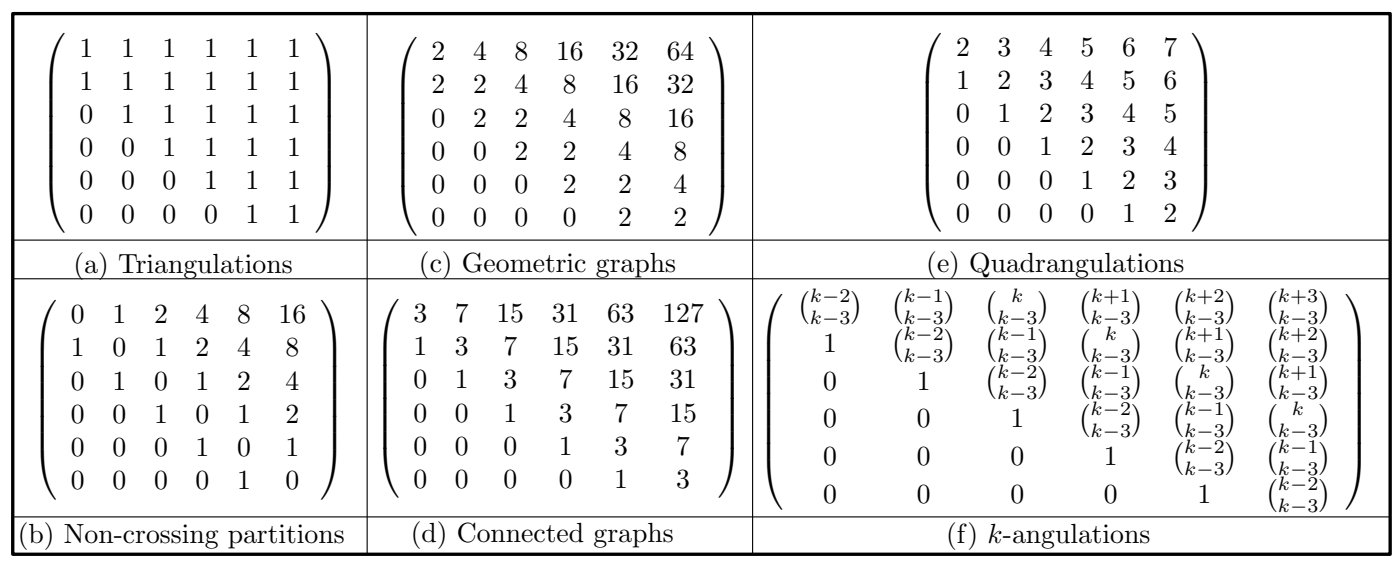

Fig. 1. Production matrices for different graph classes, for $n=6$.

our results for $k$-angulations and connected geometric graphs.

\section{Counting $k$-angulations}

A $k$-angulation is a 2-connected plane graph in which every internal face is a $k$-gon. For 3 -gons we fall into the well-known case of triangulations. The number of triangulations of a set of $n+2$ points in convex position is the Catalan number $C_{n}=\left(\begin{array}{c}2 n \\ n\end{array}\right) \frac{1}{n+1}$. In general, the number of $k$-angulations with $p k$-gons is given by $\frac{1}{(k-2) p+1}\left(\begin{array}{c}(k-1) p \\ p\end{array}\right)$. The way of calculating the entries of the production matrices is similar to the mapping used by Hurtado and Noy [9] for triangulations. We use as degree of the root vertex, the number of its incident edges. And the vector $\boldsymbol{v}^{p}$ counts $k$-angulations with $p k$-gons. Once we have the $p \times p$ production matrix of $k$-angulations $K_{p}$ we apply the methods of [10] to get a formula for the number $\boldsymbol{v}_{j}^{p}$ of $k$-angulations with $p k$-gons and with root vertex of degree $j+1$, given by the $j$-th entry of $\boldsymbol{v}^{p}$.

Theorem 2.1 Let $\boldsymbol{v}^{p}$ be the vector that counts the number of k-angulations with $p k$-gons and $n=2+(k-2) p$ vertices. The $j$-th entry of $\boldsymbol{v}^{p}$, for $j=1, \ldots, p$ is:

$$
v_{j}^{p}=\frac{j}{p}\left(\begin{array}{c}
(k-1) p-j-1 \\
p-j
\end{array}\right)
$$

To prove the theorem, we use techniques and notation from [10]. We have that the A-sequence of $K_{p}$ is $\left\{1,\left(\begin{array}{c}k-2 \\ k-3\end{array}\right),\left(\begin{array}{c}k-1 \\ k-3\end{array}\right),\left(\begin{array}{c}k \\ k-3\end{array}\right), \ldots\right\}$ with generating 
function $A(t)=\frac{1}{(1-t)^{k-2}}$, so it follows (with a shift of indices) that:

$$
\begin{array}{r}
v_{j+1}^{p+1}=\frac{j+1}{p+1}\left[t^{p-j}\right] A(t)^{p+1}=\frac{j+1}{p+1}\left[t^{p-j}\right] \sum_{\ell=0}^{\infty}\left(\begin{array}{c}
(k-2)(p+1)+\ell-1 \\
\ell
\end{array}\right) t^{\ell}= \\
=\frac{j+1}{p+1}\left(\begin{array}{c}
(k-2)(p+1)+p-j-1 \\
p-j
\end{array}\right)
\end{array}
$$

For $k=3$, a formula for the characteristic polynomial can be found in [4]. In this work, we generalize this result to all values of $k$.

Theorem 2.2 The characteristic polynomial $k_{p}(\lambda)$ of the $p \times p$ production matrix of $k$-angulations satisfies the recurrence relation

$$
k_{p}(\lambda)=\left(\left(\begin{array}{l}
k-2 \\
k-3
\end{array}\right)-\lambda\right) k_{p-1}(\lambda)+\sum_{i=2}^{p}(-1)^{i+1}\left(\begin{array}{c}
k+i-3 \\
k-3
\end{array}\right) k_{p-i}(\lambda)
$$

and the solution to the recurrence relation with initial value $k_{0}(\lambda)=1$ is

$$
k_{p}(\lambda)=\sum_{\ell=0}^{p}(-1)^{\ell}\left(\begin{array}{c}
(k-2)(\ell+1) \\
p-\ell
\end{array}\right) \lambda^{\ell}
$$

Theorem 2.3 provides a characterization of the eigenvectors of $K_{p}$ : its entries are given by the characteristic polynomials of smaller production matrices, evaluated at an eigenvalue.

Theorem 2.3 Let $x=\left(x_{p-1}, \ldots, x_{0}\right)^{\top}$ be an eigenvector for an eigenvalue $\lambda$ of the $p \times p$ production matrix of $k$-angulations. Then, for $i=1, \ldots, p-1$,

$$
x_{i}=(-1)^{i} k_{i}(\lambda) x_{0}
$$

Other properties relating the characteristic polynomials and the vector of $k$-angulations have been obtained. In this abstract we only state one for $k=4$ :

Theorem 2.4 Let $Q_{i}$ be the $i \times i$ production matrix for quadrangulations, $q_{i}$ its characteristic polynomial, $\lambda$ an eigenvalue of $Q_{p}$, and $\boldsymbol{v}^{p}$ the vector of quadrangulations with $p$ 4-gons. Then

$$
\sum_{i=1}^{p} v_{i}^{p+1} q_{i-1}(\lambda)(-1)^{i+1}=\lambda^{p}
$$




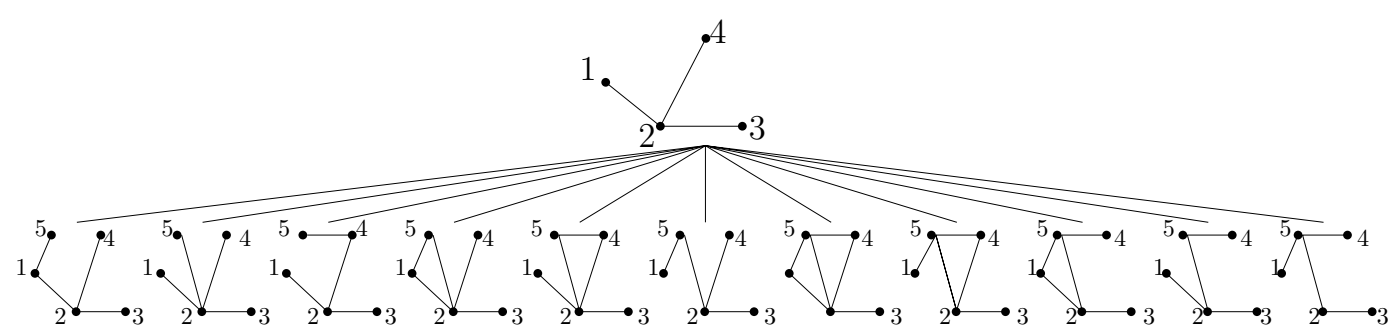

Fig. 2. The descendents of a graph in the generating tree of connected graphs. The graph has (visibility) degree 3 , and it has 1 descendent of degree 4, 3 of degree 3 , and 7 of degree 2 .

\section{Counting connected graphs}

Given a set of points in convex position $\left\{s_{1}, \ldots, s_{n}\right\}$ numbered counter - clockwise, we define the tree of connected graphs by using the following mapping between $\mathfrak{C}_{n}$ and $\mathfrak{C}_{n+1}$, where $\mathfrak{C}_{j}$ is the set of connected graphs on $j$ points. For a connected graph on $n$ points in convex position, let $F\left(s_{n+1}\right)$ be the ordered sequence of vertices visible from the point $s_{n+1}$, which is inserted between $s_{1}$ and $s_{n}$. Then we obtain a connected graph by connecting $s_{n+1}$ to all its visible vertices from $s_{i}$ to $s_{j}$ where $s_{i}, s_{j} \in F\left(s_{n+1}\right)$ for each $i \leq j$, and possibly removing the edges whose endpoints are between $s_{i}$ and $s_{j}$ in $F\left(s_{n+1}\right)$, maintaining connectivity. Figure 2 shows an example. Observe that each connected graph is generated exactly once. And these rules result into matrix (d) of Fig.1.

We point out that for connected graphs on $n$ vertices, the degree of the root vertex $s_{n}$ is defined as the number of vertices visible from $s_{n+1}$.

We have obtained analogous theorems as for $k$-angulations, see Fig. 3 . The proofs are similar to the ones for $k$-angulations: applying results on Riordan arrays and induction.

\begin{tabular}{|c|c|}
\hline $\begin{array}{c}\text { Vector } v_{k}^{n} \text { of } \\
\text { connected graphs }\end{array}$ & $\frac{k}{n-1} 2^{n-1} \sum_{j=0}^{n-1}\left(\begin{array}{c}n-1 \\
j\end{array}\right)\left(-\frac{1}{2}\right)^{j} \sum_{\ell=0}^{n-k-1}\left(\begin{array}{c}n-2-j+\ell \\
\ell\end{array}\right)\left(\begin{array}{c}j+n-\ell-k-2 \\
n-\ell-k-1\end{array}\right) 2^{\ell}$ \\
\hline $\begin{array}{c}\text { Characteristic } \\
\text { polynomial } c_{n}(\lambda)\end{array}$ & $\sum_{t=0}^{n}\left(\sum_{\ell=0}^{t}\left(\begin{array}{c}t \\
\ell\end{array}\right)(-1)^{t} 2^{t-\ell} 3^{n+2 \ell-3 t-2}\left[2\left(\begin{array}{c}\ell \\
n+2 \ell-3 t-2\end{array}\right)+9\left(\begin{array}{c}\ell+1 \\
n+2 \ell-3 t\end{array}\right)\right]\right) \lambda^{t}$ \\
\hline Eigenvector & $\left\{\left(x=\left(x_{n-1}, x_{n-2}, \cdots, x_{0}\right) \mid x_{i}=(-1)^{i} c_{i}(\lambda) x_{0}\right\}\right.$ \\
\hline
\end{tabular}

Fig. 3. Results obtained for connected graphs, where $n$ is the number of vertices, and $k$ the degree of the root, defined and based on visibility. 


\section{Acknowledgments}

This project has received funding from the European Union's Horizon 2020 research and innovation programme under the Marie Skłodowska-Curie grant agreement No 734922. C. H. and R. I. S. were supported by MINECO MTM2015-63791-R and Gen. Cat. 2017SGR1336 and 2017SGR1640. R. I. S. was also supported by MINECO through the Ramón y Cajal program.

\section{References}

[1] E. Barcucci, A. D. Lungo, E. Pergola, and R. Pinzani. ECO: a methodology for the enumeration of combinatorial objects. J. Differ. Equations Appl., 5(45):435-490, 1999.

[2] E. Deutsch, L. Ferrari, and S. Rinaldi. Production matrices. Adv. in Appl. Math., 34(1):101-122, 2005.

[3] E. Deutsch and L. Shapiro. A survey of the Fine numbers. Discrete Mathematics, 241:241-265, 2001.

[4] G. Fairweather. On the eigenvalues and eigenvectors of a class of Hessenberg matrices. SIAM Rev., 13(2):220-221, 1971.

[5] P. Flajolet and M. Noy. Analytic combinatorics of non-crossing configurations. Discrete Mathematics, 204(1-3):203-229, 1999.

[6] C. Huemer, A. Pilz, C. Seara, and R. I. Silveira. Production matrices for geometric graphs. Electr. Notes Discrete Math., 54:301-306, 2016.

[7] C. Huemer, A. Pilz, C. Seara, and R. I. Silveira. Characteristic polynomials of production matrices for geometric graphs. Electr. Notes Discrete Math., 61:631-637, 2017.

[8] C. Huemer, A. Pilz, and R. I. Silveira. A new lower bound on the maximum number of plane graphs using production matrices. In Abstracts 34th European Workshop on Computational Geometry, pages 9:1-9:6, 2018.

[9] F. Hurtado and M. Noy. Graph of triangulations of a convex polygon and tree of triangulations. Comput. Geom., 13(3):179-188, 1999.

[10] D. Merlini and M. C. Verri. Generating trees and proper Riordan arrays. Discrete Math., 218(1-3):167-183, 2000. 